\title{
Strategic Competence: Bridging The Cross-Cultural Communication
}

\section{Lilla Musyahda}

Airlangga University

\begin{abstract}
The writer of this article is interested in examining the strategic competence, i.e., the ability to solve communication problems an inadequate command of the linguistic and sociocultural code because it, can contribute to the development of an overall communicative competence. The concept of strategic competence is within the general framework of interlanguage development. The article describes two basic types of communication strategies: reduction and achievement, concentrating particularly on the use of achievement strategies at the discourse level for English learners to manage a communication across culture appropriately.
\end{abstract}

Key words: strategic competence, interlanguage pragmatics, crosscultural communication

The definition of communication strategies, coined by Larry Selinker (in Ellis, 1985:180), is mentioned by Stern (1983:411), i.e., techniques of coping with difficulties in communicating in an imperfectly known second language. Terrel in Jack C. Richard (1983:11) states that communication strategies are also crucial at the beginning stages of second language learners. For example, when one does not know the English term 'railway station', she might try to paraphrase it into as 'the place where trains go' or 'the place for train'. Canale and Swain in Rod Ellis (1985:184) proposes that communication strategies are to be seen as a part of communicative competence and identifies it as "strategic competence" which means "how to cope in an authentic communicative situation and how to keep the communicative channel open". To sum up Communication strategies can defined as psycholin- 
guistic plans, which exist as part of the language user's communicative competence. They are potentially conscious and serve as substitutes for production plans, which the learner is unable to implement (Ellis, 1985:180).

Hence it has two main points, that is, conscious and productive. Bachman (1996:70) reinforces the concept of strategic competence as a set of metacognitive components, or strategies, which can be thought of as higher order executive processes that provide a cognitive management function in language use, as well as in other cognitive activities.

The processes will form the interplay between two languages. They lead to interesting phenomena in which many aspects are involved. Language is the chief means by which people communicate, yet simply knowing the words and grammar of a language does not ensure successful communication. Its interpretation depends on a multiplicity of factors, including familiarity with the context, intonational clues and cultural assumption. Phenomena like these are the concern of pragmatics, Blum-Kulka in Van Dijk proposes the formal definition of pragmatics.

Pragmatics is the science of language seen in relation to its users (Mey, 1993:5); in other words, the focus of pragmatics is on both the processes and the product of communication, including its cultural embeddedness and social consequences (Van Dijk, 1997:37).

As both of the processes and the product of communication are the main concern of this study, Chomsky's view on pragmatics cited from Mey (1993:36) also reinforces that:

...... The proper domain of pragmatics would be what Chomsky has called performance, that is to say, the way the individual user went about using his or her language in everyday life. The practice of performance would be then defined in contrast to the user's abstract competence, understood as his or her knowledge of the language and its rules

Blum-Kulka in Van Dijk (1997:43) defines interlanguage pragmatics as a study which concerns with the acquisition and performance of pragmatic skills in a second language. Acquisition is the representative of competence; therefore the writer is interested to examine how strategic competence-the ability to solve communication problems despite an inadequate command of the linguistic and sociocultural code-can contribute to the development of an overall communicative competence.
The development of communicative competence consists of (a) organisation competence and (b) pragmatic competence. Organisation competence refers to ability to form a formal structure of a language and produce correct grammatical sentences or it comprises knowledge of linguistic units and the rules of joining them together at the levels of sentence (grammatical competence) and discourse (textual competence). Respectively, the competence is divided into two, that is, grammatical and textual. Grammatical competence covers vocabulary, morphology, syntax and phonology; Textual competence refers to the coherent and rhetoric.

Pragmatic competence defines the rational side of mind. Individuals lacking this competence would be unable to utter relevant arguments or even to form relevant thoughts. There has never been a report on the existence of a culture on earth in which normal individuals would be lacking the ability to be relevant.

Pragmatic ability in a second or foreign language is part of a nonnative speakers (NNS) communicative competence and therefore has to be located in a model of communicative ability. Pragmatic competence subdivides into 'illocutionary competence' and 'sociolinguistic competence'. 'Illocutionary competence' can be glossed as 'knowledge of communicative action and how to carry it out'. The term 'communicative action' is often more accurate than the more familiar term 'speech act' because communicative action is neutral between the spoken and written mode, and the term acknowledges the fact that communicative action can also be implemented by silence or nonverbally. 'Sociolinguistic competence' comprises the ability to use language appropriately according to context. It thus includes the ability to select communicative acts and appropriate strategies to implement them depending on the current status of the 'conversational contract' (Richard, 1983:1)

Oller in Bachman (Bachman, 1996:70) points out that strategic competence refers to the mastery of verbal strategy and non- verbal strategy to overcome the failure in communication due to inaccurate competence. Thus, strategic competence consists of three components, that is, (a) assessment, (b) planning, and (c) execution. Assessment covers the area of information recognition, including language variety or dialect, determining language competence to achieve the goal of effective communication, and evaluating the purpose of in-progress communication. Planning consists of relevante point of language competence, that is, grammatical, textual, illocutionary and a formula to achieve the goal of communication. Execution is relevance 
with the mechanism of psychophisilogic to carry out the planning relates to the context and purpose of commonication.

Basically, psychophisiologic mechanism is a neurologic and physiologic process involved in phases of language use. As language activity is able to operate the model of productive and /or receptive, different skills are needed for each activity. At the receptive process, the skills of auditory and visual have important roles, while productive aspect involves the skills of neuromuscular.

Stern (1983:229) proposes that the third element of communicative competence besides grammatical and sociolinguistic competence, Strategic Competence, is needed by the language learner to know how to conduct himself as someone whose sociocultural and grammatical competence is limited, i.e., to know how to be a 'foreigner'.

\section{STRATEGIC COMPETENCE IN INTERLANGUAGE DEVELOP- MENT}

The notion 'interlanguage' alludes to a language "between" two languages, i.e. a target language (TL) norm that a student is trying to achieve, and his first language (L1). The interlanguage has characteristics of both of these languages. However, the nature of the blending, or how "between" is to be interpreted has always been vague in second language acquisition (SLA).

The main features of interlanguage, which will be used in the diagnostic system, are overgeneralisation of TL rule statements and transfer from $\mathrm{L} 1$. In the diagnostic system, overgeneralisation will be implemented as constraint relaxation and transfer will be implemented by means of an alternative L1 based grammar. Transfer is understood in the sense, which is used in SLA research.

The second language learner, at the beginning of his study, has his attention focuse on one norm of the language whose sentences he is attempting to produce. The sketch of process can be described as follows: (1) accepting the notion of target language (TL), i.e., the second language learner is attempting to learn, which means that there is only one norm of the dialect within the interlingual focus of attention of the learner; (2) focusing on analytical attention on interlanguage data (IL), i.e. the utterances that are produced when the learner attempts to say sentences of a TL. The set of utterances for most learners of a second language is not identical to the hypothe- sized corresponding set of utterances which are produced by native speakers of the TL had he attempted to express the same meaning as the learner.

As the two sets of utterances are not identical, it could be relevant to the theory of second language learning, that is, one would be completely justified in hypothesizing perhaps even compelled to hypothesize, the existence of a separate linguistic system based on the observable output which results from learner's attempted production of a TL norm. It is assumed that this linguistic system is called interlanguage.

Strategic competence in the frame of interlanguage respectively can be assumed as the ability to cope with unexpected problems, when the speaker of foreign language has no ready-made solution available. It entails to certain phenomenon in coping with the obstacles, which can be measured using general parameter of oral production. Mariani (1994) proposes "reduction strategies" and "achievement strategies". The former strategies can affect the following circumstances:

a. Content : -topic avoidance -message abandonment -meaning replacement

b. Modality (politeness makers)

c. Speech act (communicative action) namely:

The achievement strategies will influence two areas respectively,

1. Words/sentence level:

a. Borrowing (code-switching)

b. Foreignizing

c. Literal translation

d. Interlanguage-based: -Generalization

-Paraphrase

-Restructuring

2. Discourse level:

a. Opening and closing conversation

b. Keeping a conversation going

c. Expressing feeling and attitude

d. Managing interaction (handling a topic/discussion)

e. Negotiating meaning and intention 
The Typology of Interlanguage Stages

IDEAL ZERO COMPETENCE

IDEAL NATIVE SPEAKER

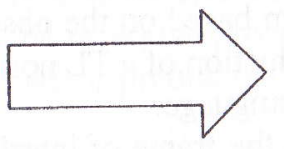

\section{INTERLANGUAGE STAGES}

(Mariani, 1994)

\section{THEORY OF CROSS-CULTURAL PRAGMATICS}

To understand human interaction we have to comprehend 'interactional' meanings expressed in speech. Unfortunately, there are many aspects involved in this interaction, as consequently a failure will come up in describing the messages. The goal of a communication is to transfer message from the speaker to the hearer. As describing meanings part of the process of understanding utterances, the hearers perceive it in their own value. Meanwhile, there is still a gap between describing a meaning in LI and L2 respectively.

At one time, a versatile instrument to describe a meaning is standard lexicographic descriptions of words such as question and interrogative. However, it is a general description of precision and a vague boundary for both of the words. For example, Webster thesaurus and dictionary explanation for such words;

Question - a command or interrogative expression used to elicit

Interrogative - an interrogative utterance, a question

All such explanations of interactional meanings are clearly, totally circular (Wierzbicka, 1991:6). Furthermore, they are defined in terms which is language specific (usually, English specific) and which provide no languageindependent, universal perspective on the meanings expressed in linguistic interaction.
In the meantime, the process of interaction between the speakers and hearers take place in the local circumstances in which universal values of such activities are not equal. The phenomenon has emerged in the last decade that shows a growing reaction against this of misguided universalism. It leads to the transpiring of a new direction in language study associated with the term 'cross-cultural pragmatics'. The main ideas, which have informed this new direction in the study of language, are these: (Wierzbicka, 1991:69)

(1) In different societies, and different communities, people speak differently.

(2) These differences in ways of speaking are profound and systematic.

(3) These differences reflect different cultural values, or at least different hierarchies of value.

(4) Different ways of speaking, different communicative styles can be explained and made sense off, in terms of independently established different cultural values and cultural priorities.

\section{CONCLUSION}

The strategic competence strategies basically relates to reduction strategies and achievement strategies. Reduction strategies can affect (a) content, which can be in the form of topic avoidance, massage abandonment, meaning replacement; (b) modality or politeness makers; and (c) speech act (communicative action). Meanwhile, achievement strategies influence two areas that is, words/sentence level and discourse level. At words/sentence level, the strategies are in the function of borrowing/code-switching, foreignizing, literal translation, and interlanguage-based in the form of generalization, paraphrasing, and restructuring. At Discourse level the strategies can take the form of opening and closing conversation, keeping conversation going, expressing feeling and attitude, managing attitude and negotiating meaning and attention.

What is found in an EFL context are reduction strategies which consist of content and modality, whereas speech act or communicative action is not found since the context is of a non-formal situation. Furthermore, the writer finds achievement strategies in the form of borrowing(code-switching)and interlanguage based which can be 
seen as paraphrasing and restructuring.

The communication across culture may become less frustrating if we know that different communicative styles, and different norms of social interaction are not only universal but also simple and easy to understand for the second language learners. It can be hoped that the kind of competence can be used as a tool for investigating linguistic interaction in different cultural settings. Particularly, it can be as a basis for teaching successful cross-cultural communication.

\section{REFERENCES}

Bachman, Lyle F. 1997. Language testing in Practice: designing and Developing Useful Language Test. Hongkong: Oxford.

Ellis, Rod. 1985. Understanding Second Language Acquisition. Oxford: Oxford University Press.

Ellis, Rod. 2000.Second Language Acquisition. Oxford: OUP.

Richards, Jack C. 1983. Language and Communication. London: Longman.

Mariani, Luciano.1994. The Typology of Interlanguage Strages. TESOL Journal, June 1994. http://web.tiscali.it/learningpaths/ $\underline{\mathrm{htm}}$

Mey, Jacob L. 1993. Pragmatics An Introduction. London: Blackwell. Stren, H.H. 1983. Fundamental Concepts of Language Teaching. Oxford: Oxford University Press.

Van Dijk, Teun A. 1997. Discourse as Social Interaction.London: Sage Publication

Wierzbicka, Anna. 1991. Cross-Cultural Pragmatics The Semantics of Human Interaction.Berlin: Mouton De Gruyter. 